\title{
Multimodal Interactive Kiosk System for Diabetes Education and Diagnosis in Rural India
}

\author{
N.T.Ajith \\ Department of Information \\ Technology \\ Sri Krishna College of Engineering \\ and Technology, Coimbatore \\ Tamil Nadu, India
}

\author{
Lakshman $\mathrm{R}$ \\ Department of Information \\ Technology \\ Sri Krishna College of Engineering \\ and Technology, Coimbatore \\ Tamil Nadu, India
}

\author{
Shankar S \\ Assistant Professor \\ Department of Information \\ Technology \\ Sri Krishna College of Engineering \\ and Technology, Coimbatore \\ Tamil Nadu, India
}

\begin{abstract}
In this paper, we present a framework for a multimodal interactive rural kiosk for spreading awareness about diabetes and monitoring of blood glucose level, thus aiding its diagnosis in rural India. There were an estimated 40 million persons with diabetes in India in 2007 and this number is predicted to rise to almost 70 million people by 2025 . In the last 15 years the prevalence of Diabetes in rural India has risen from 2.4 per cent to 6.4 per cent of the total population. In rural India ,there is a lack of awareness about Diabetes. Primary Health Centers(PHC) are the prime caterers here to provide basic healthcare services which also includes diagnosis and treatment for Diabetes. But, in the current scenario, each Primary Health Center covers a population of 1,00,000 and spread over about 100 villages[5]. This is not sufficient for the population and access becomes difficult due to spatial constraints. An interactive kiosk is a computer terminal that provides information access via electronic methods. Multimodal interaction provides the user with multiple modes of interfacing with a system. Our proposed Multimodal Interactive Kiosk System (MIKS) provides easy access and good communication to create awareness and also diagnoses Diabetes. The blood glucose level is monitored using a glucometer which is interfaced via USB with the kiosk. Telemedicine facilities are also provided to the users if they are diagnosed with Diabetes so that they can decide the further course of action. The communication can be done by mechanical backhaul[6] for low cost and high efficiency.
\end{abstract}

\section{Categories and Subject Descriptors}

\section{General Terms \\ Design}

\section{Keywords}

Multimodal interactive kiosk, Diabetes Type 2, Telemedicine

\section{INTRODUCTION}

Diabetes has emerged as a major healthcare problem in India. There were an estimated 40 million persons with diabetes in India in 2007 and this number is predicted to rise to almost 70 million people by 2025 . It is estimated that every fifth person with diabetes will be an Indian. Due to these sheer numbers, the economic burden due to Diabetes in India is amongst the highest in the world. The real burden of the disease is however due to its associated complications which lead to increased morbidity and mortality. The transition from a traditional to modern lifestyle, consumption of diets rich in fat and calories combined with a high level of mental stress has compounded the problem further. There are several studies from various parts of India which reveal a rising trend in the prevalence of Type 2 Diabetes in the urban areas.

The recent data has illustrated the impact of socio-economic transition occurring in rural India. The transition has occurred in the last 15 years and the prevalence has risen from 2.4 per cent to 6.4 per cent. There are many varieties of Diabetes, most notable being insulin dependent or Type 1 and non-insulin dependent or Type 2 diabetes. Type 1 Diabetes is an immunological disease, with a genetic background. Type 1 Diabetes occurs mainly in children. Type 2 Diabetes is primarily a hereditary disease, which is precipitated by environmental factors like lack of proper diet and lack of exercise. In India, most Diabetics( about 95\%) belong to Type 2. Type 2 Diabetics may have a partial insulin deficiency. So the measurement of blood glucose level is a primary means of diagnosing Diabetes.

In rural India ,there is a lack of awareness about Diabetes and access to healthcare facilities is cumbersome. Multimodal interfaces require effective parsing and understanding of utterances whose content is distributed across multiple input modes [9]. Therefore, we propose a Multimodal Interactive Kiosk System to provide awareness and diagnose Diabetes. Integration of technology allows kiosks to perform a wide range of functions, evolving into self-service kiosks. An interactive kiosk can be used by a person with minimal technical knowled ge.

A multimodal kiosk provides different modes such as speech, touch screen etc for interacting with the user. Automatic teller machines and other simple interactive computer systems are used increasingly in the modern society [7]. Dialogs were significantly shorter with the kiosk interface than with the telephony interface, and users preferred the kiosk interface and found it easier to use [8]. Our proposed system provides awareness by explaining what diabetes exactly is, its causes, the symptoms of diabetes, its risk factors, its various types and other related details. It also diagnoses Diabetes by analyzing the blood glucose level from the user.

\section{RELATED WORK}

Kiosks can be categorized depending on their purpose. Borchers et al. [1] defines four such categories: information kiosks, advertising kiosks, service kiosks and entertainment kiosks. However, these categories overlap somewhat. 
Touch-screens are typically used in kiosks because they provide an easy way to interact for people who have little or no experience in computers. Other modalities such as vision and speech can also help improve this interaction. For example, Lamel et al. [2] describe a service kiosk that includes a speech recognition component.

Multimodal kiosks can be more effective as an interactive tool due to its several modes of interaction with users. For example, makinen et al [3] deploy a multimodal information kiosk is discussed for museum. A talking face is used for better interaction.

Telemedicine can be a powerful tool after the diagnosis of Diabetes. The user can have the expert's ideas on his/her disease and can be referred for further course of action. Sameer S Sawarkar[4] et al has discussed the use of telemedicine as a suitable health care tool for rural India.

A.Seth et al [6] describe a reliable and low cost communication architecture for Rural kiosks.

\section{OUR PROPOSED FRAMEWORK}

\subsection{Components}

\subsubsection{Interactive Screen}

The interactive screen can be used by the user to obtain information regarding Diabetes. The interactive screen responds to touch akin to the ATM used by banks. The operation of the interactive screen requires no technical knowledge.

The $3 \mathrm{D}$ avatar is used to make better understanding through voice explanation. The avatar faces the user based on the input from the web cam by the concept of face recognition.

A virtual keyboard is displayed by which the user can enter the details.

\subsubsection{Web Camera}

The web camera is used to capture the image of the subject. The web camera also facilitates the telemedicine process. It can be used for the multimodal access of the kiosk[2]. Based on the input from the web cam, the avatar is made to face the user for better communication.

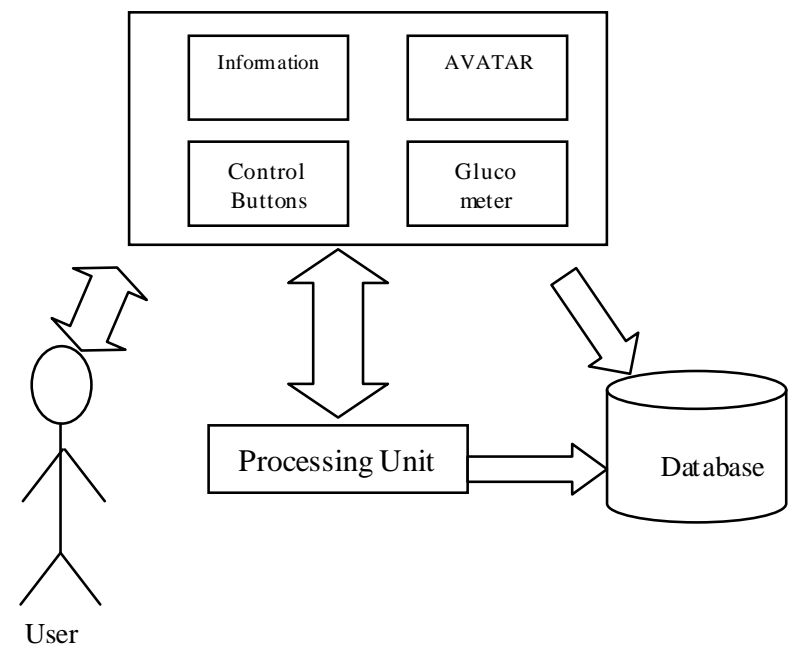

Figure 1. Framework of proposed system

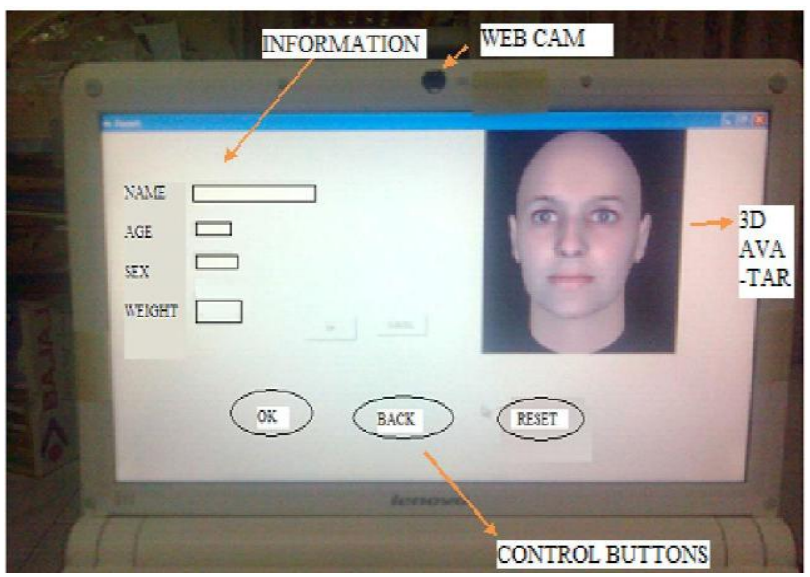

Figure 2. Interactive Screen

\subsubsection{Database}

The database is used to store the information about patients. The database records the name, age, sex, reading of the blood sugar level from the glucometer. The database consists of the standard literature about acceptable values of blood glucose levels specific for the age group and gender so that the comparison with measured values can aid in the diagnosis of diabetes

\subsubsection{Processing Unit}

The processing unit, which is a part of the database is used for processing the data obtained from the user. It matches the acquired reading from the integrated glucometer against the literature from database. The result is then displayed to the user via the interactive screen.

\subsubsection{Glucometer}

The glucometer is a device used to calculate the amount of blood sugar level present in the human body. Modern glucometers are simple with a LCD display. The user needs to place a drop of blood in the strip provided in the glucometer using a lancet. The glucometer evaluates and displays the result in less than 20 seconds. The necessary guidelines are pictorially represented through the Interactive screen.

\subsubsection{Internet Connection}

The multimodal interactive kiosk is also provided with internet connection by which it is connected to a major city hospital. This helps in updating the information about Diabetes by the doctors. It also helps in acquiring the telemedicine facilities. The communication can be done by mechanical backhaul[6] for low cost and high efficiency.

\subsubsection{Telemedicine Facility}

In telemedicine method, the user gets to interact with a doctor of an esteemed hospital with the help of internet. Provisions are done such that the doctors can view the information about user from the database during interaction. Also, links to esteemed hospitals are given so that the user can book an appointment.

\subsubsection{Keyboard}

The keyboard consists of specialized keys for control purposes. The various keys include, a OK key, a CANCEL key, and a BACK key. This can provide a tactile mode of interaction for the user. 


\subsection{The Process}

The user enters the kiosk centre. The attached web cam views him and makes a steady output. The initial interactive screen welcomes him and asks him a choice whether he needs to get details of the disease or he wants to diagnose for diabetes. The user makes an appropriate choice.

If the user chooses to know details about Diabetes, the screen gives him information about Diabetes from the database. The screen which displays the symptoms has a link to the diagnosis screen which facilitates the user to diagnose the disease if he finds the provided symptoms. The stored information is updated periodically by a group of selected experts through the internet.

If the user chooses diagnosis of diabetes, the specific details of the user are got from the user. These information are stored in a database for future reference. Then, the attached glucometer is used to measure the blood glucose level. For this purpose, the user is requested to place a drop of blood in the strip provided with the glucometer with the help of a lancet. The whole process is pictorially explained through the interactive screen. The glucometer performs the necessary tests, finds the blood glucose level and sends the measurement to the process unit. The process unit compares the measured value with the standard literature and decides whether the user has diabetes. This result along with the previously stored personal information is printed with the help of an attached printer.

The printout which consists of the user's name, sex, weight, present sugar level and the assessment of the process unit can be used for consultation in the hospital.

Facilities for telemedicine are also provided in the system. In telemedicine method, the user gets to interact with a doctor of an esteemed hospital with the help of internet. Provisions are done such that the doctors can view the information about user from the database during interaction. Also, links to esteemed hospitals are given so that the user can book an appointment.

\section{OPERATION OF THE MIKS AS AN AWARENESS TOOL}

\subsection{Description of the Operational Steps}

1. The user sends a request for the awareness information by choosing appropriate options through interactive screen.

2. Create awareness to the users through stored content.

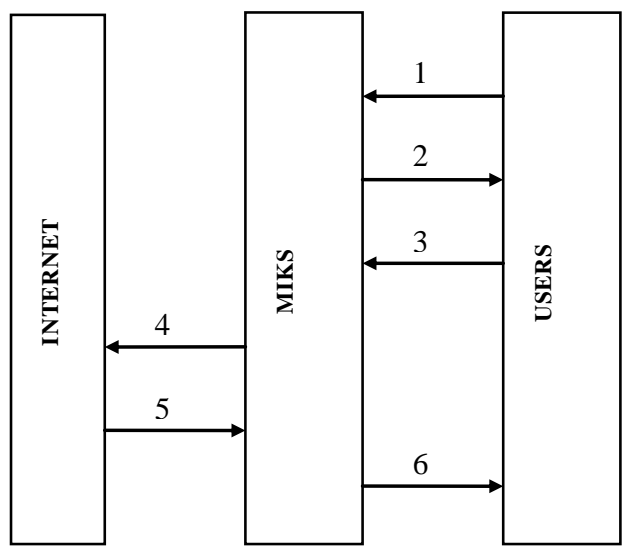

Figure 3. Sequence of operations of MIKS as an Awareness tool.
3. Request more information through the MIKS

4. Send appropriate request to the Internet.

5. Response from the Internet to MIKS.

6. Provide users with the expert information from internet.

The above illustration gives an overall view of the operation of the MIKS as an awareness tool. The sequence of various operations involved in this mode of operation of the MIKS is also clearly described. If the user chooses to know details about diabetes, the screen gives him information about what diabetes exactly is, the symptoms of diabetes, its risk factors, its various types and other related details. The screen which displays the symptoms has a link to the diagnosis screen which facilitates the user to diagnose the disease if he finds the provided symptoms. And finally, all updated information can be viewed from a website on request from the user through the internet. The website can be accessed by a group of expert doctors.I

\subsection{Awareness about the Symptoms}

Various symptoms of Diabetes can be used to decide whether a person is suffering from Diabetes. Several symptoms such as Diabetic retinopathy, Renal (kidney) disease, Tingling, burning, numbness, tightness, shooting or stabbing pain in the hands, feet or other parts of body, autonomic neuropathy, scanty or profuse sweating, difficulty of sensing when your bladder is full, increased sexual problems, weakness, dizziness, and fainting, Chest pain (angina) or shortness of breath are discussed in detail. The chances of a heart attack or stroke are also discussed. If any of these symptoms occur, the user has a facility to move to diagnosis.

\subsection{A wareness about the Risk factors}

The risk factors of any disease can be very useful in prevention of the disease. This concept is no different for Diabetes. The main risk factors such as Obesity, Sedentary Lifestyle, Unhealthy Eating Habits, Family History and Genetics, Increased Age, High Blood Pressure and High Cholesterol, History of Gestational Diabetes are all discussed in detail. This helps the users in prevention and simultaneously creates awareness of Diabetes.

\section{OPERATION OF MIKS AS A DIAGNOSTIC TOOL}

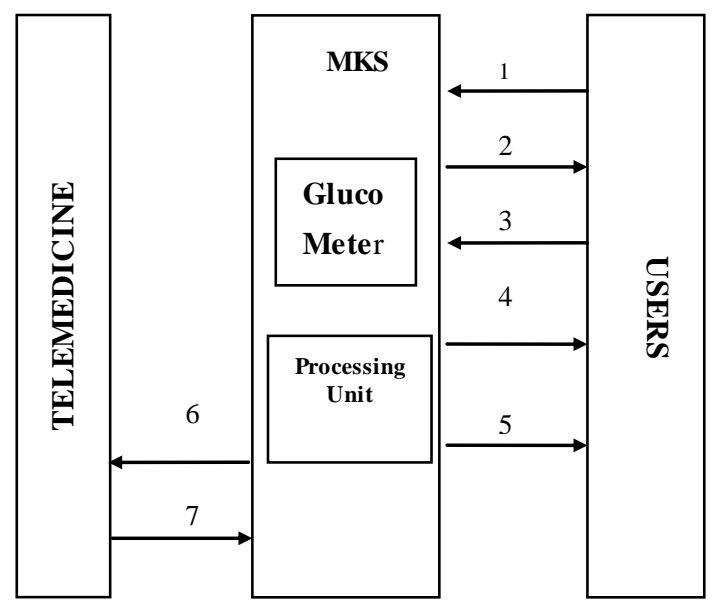

Figure 4. Sequence of operations of MIKS as a Diagnostic tool. 
1. The user selects the diagnose option from the interactive screen of the MIKS The MIKS asks the user to enter his/her details

2. User uses the lancet and places a drop of blood in the glu cometer strip

3. The glu cometer analyzes the blood sample and provides the result to the processing unit. The processing unit compares the sample result obtained with the literature and displays via the interactive screen.

4. The result is also dispatched by means of a printed format and given to the user.

5. The user requests the telemedicine facility if diagnosed with diabetes.

6. The experts interact with the user through the telemedicine facility.

The above illustration gives an overall view of the operation of MIKS as a diagnostic tool. The sequence of various operations involved in this mode is also clearly described. In this mode of operation, the interaction is between the user and the MIKS. The details entered by the user are recorded into the database. The processing unit in our model contains the standard literature which consists of the classification of blood sugar levels for the average adult. The MIKS has a voice interactive guide(VIG) in the form of an Avatar.

In telemedicine method, the user gets to interact with a doctor of an esteemed hospital with the help of internet. Provisions are done such that the doctors can view the information about user from the database during interaction. Also, links to esteemed hospitals are given so that the user can book an appointment.

\section{FUTURE WORK}

Our proposed system uses three modes of interactions namely the touch screen for touch, the speech from the 3D avatar, and through keys in a keyboard with specialized keys corresponding to the various options. This can be extended to using voice recognition so that the kiosk can respond to the several requests and queries of the user.

The work on Diabetes can also be extended to other major diseases affecting the Rural population.

\section{CONCLUSION}

The proposed multimodal interactive kiosk system for diabetes awareness program ensures that the people in rural India are able to access the system with maximum convenience. The operational modes of the multimodal kiosk system as a tool for diagnostic tool is also clearly described. The system will help the government in disseminating the information about Diabetes to the rural population.Thus, the MIKS helps in prevention of this disease. In future, the MIKS can be extended to use the cellular system services efficiently. MIKS can be enhanced to be interoperable with other peripheral devices like PDA and smart phones to which the glucometer reading can be downloaded.

\section{REFERENCES}

[1] Borchers J., Deussen O. and Knörzer C., Getting It Across: Layout Issues for Kiosk Systems. In: Proceedings of the Workshop on W3-Based OnlineKiosk Systems, Third International World-Wide Web Conference, Darmstadt 1995. Reprinted in: SIGCHIBulletin 27, 4 (October 1995), 68-74.

[2] Lamel, L., Bennacef, S., Gauvain, J. L., Dartiguest, H.and Temem J. N.: User Evaluation of the Mask Kiosk, ICSLP '98, Sydney, Australia (1998).

[3] Erno Mäkinen, Saija Patomäki, and Roope Raisamo : Experiences on a Multimodal Information Kiosk with an Interactive Agent, NordiCHI, October 19-23, 2002

[4] Sameer S Sawarkar, Rajeev Kumar, Manish Ingle, Dr. C. S. Sukumar, R. Anuradha: Kiosk-based Scalable and Selfsustainable Telemedicine Solution for Rural India, Global-ehealth, 2006.

[5] Government services of India, http://india. gov.in/citizen/health

[6] A. Seth, D. Kroeker, M. Zaharia, S. Guo, and S. Keshav: Lowcost Communication for Rural Internet Kiosks Using Mechanical Backhaul Proceedings of the 12th annual international conference on Mobile computing and networking, Pages: 334 - 345,2006

[7] R. Raisamo, A Multimodal User Interface for Public

[8] Information Kiosks. In Proceedings of PUI Workshop, San

[9] Francisco, 1998

[10] S. Naray anan, G. DiFabbrizio, C. Kamm, J. Hubbell, B. Buntschuh, P. Ruscitti, and J. Wright. 2000. Effects of Dialog Initiative and Multi-modal Presentation Strategies on Large Directory Information Access. In Proceedings of ICSLP 2000, pages 636-639.

[11] M. Johnston and S. Bangalore. 2000. Finite state Multimodal Parsing and Understanding. In Proceedings of COLING 2000, pages 369-375, Saarbr"ucken, Germany. 